5. Bartels Bartels MD, Larner-Svensson H, Meiniche H, et al. Monitoring methicillin-resistant Staphylococcus aureus and its spread in Copenhagen, Denmark, 2013, through routine whole-genome sequencing. Euro Surveill 2015;20. doi: 10.2807/1560-7917.es2015.20.17.21112.

6. Uelze L, Grützke J, Borowiak M, Hammerl JA, Juraschek K, Deneke C, Tausch SH, Malorny B. Typing methods based on whole genome sequencing data. One Health Outlook 2020;2. doi.org/10.1186/s42522-020-0010-1.

7. Bartels MD, Worning P, Andersen LP, et al. Repeated introduction and spread of the MRSA clone t304/ST6 in northern Europe. Clin Microbiol Infect 2020. doi: 10.1016/j.cmi.2020.05.004.
8. Cunningham SA, Jeraldo PR, Schuetz AN, Heitman AA, Patel R. Staphylococcus aureus whole genome sequence-based susceptibility and resistance prediction using a clinically amenable workflow. Diagn Microbiol Infect Dis 2020 97:115060.

9. Enström J, Fröding I, Giske CG, et al. USA300 methicillin-resistant Staphylococcus aureus in Stockholm, Sweden, from 2008 to 2016. PLoS One 2018;13:e0205761.

10. Giske CG, Dyrkell F, Arnellos D, et al. Transmission events and antimicrobial susceptibilities of methicillin-resistant Staphylococcus argenteus in Stockholm. Clin Microbiol Infect 2019;25:1289.e5-1289.e8.

\title{
Neosartorya hiratsukae: Environmental isolation from intensive care units in an Italian hospital
}

\author{
Anna Prigitano $\mathrm{PhD}^{1}$ (1), Maria C. Esposto $\mathrm{BS}^{1}$, Davide Carnevali $\mathrm{MD}^{2}$, Emanuele Catena $\mathrm{MD}^{3}$, \\ Francesco Auxilia MD, $\mathrm{PROF}^{1,4}$, Silvana Castaldi MD, PROF ${ }^{1,5, a}$ and Luisa Romanò PhD, PROF ${ }^{1, a}$ \\ ${ }^{1}$ Department of Biomedical Sciences for Health, Università degli Studi di Milano, Milano, Italy, ${ }^{2}$ Postgraduate School in Public Health, Department of Biomedical \\ Sciences for Health, Università degli Studi di Milano, Milano, Italy, ${ }^{3}$ Department of Anaesthesiology and Intensive Care Unit, Luigi Sacco Hospital, Milano, Italy, \\ ${ }^{4}$ ASST Fatebenefratelli Sacco, Milano, Italy and ${ }^{5}$ Fondazione IRCCS Ca' Granda Ospedale Maggiore Policlinico di Milano, Italy
}

To the Editor-Fungi are opportunistic pathogens, ubiquitously distributed. Fungal colonization acquired from the environment might evolve into an invasive infection during hospitalization due to immunosuppressive treatments to which patients are exposed. Environmental surveillance in hospital settings requires special attention to prevent fungal and non-fungal infections. Invasive aspergillosis is one of the most important infections caused by Aspergillus. Neosartorya hiratsukae, teleomorph of the Aspergillus section Fumigati, is a rare fungus isolated for the first time in Japan from indoor air and from pasteurized aloe juice. ${ }^{1}$

Neosartorya hiratsukae is an opportunistic pathogen and only few cases of human infections have been reported worldwide. ${ }^{2-6}$ $N$. hiratsukae is often misidentified because it cannot be distinguished from A. fumigatus sensu stricto by conventional morphological macroscopic and microscopic analyses or by the extensively used matrix-assisted laser desorption/ionization time-of-flight mass spectrometry (MALDI-TOF-MS). However, rapid and accurate identification is important for therapeutic purposes due to the different patterns of susceptibility to antifungal drugs.

In this study, we investigated the environmental isolation of $N$. hiratsukae from 2 different intensive care units (ICUs) in a hospital in Milan, Italy. In January 2020, environmental sampling was conduted using contact plates as part of an environmental surveillance study. In total, 9 bed stations and 2 medical stations were sampled, for a total of 55 samples. After macroscopic and microscopic analyses, identification was confirmed by molecular analysis. Antifungal susceptibility testing was performed by broth microdilution assay according to European Committee on Antimicrobial Susceptibility Testing (EUCAST) to determine the minimum inhibitory concentration (MIC). ${ }^{7}$ The following

Author for correspondence: Dr Anna Prigitano, E-mail: anna.prigitano@unimi.it aAuthors of equal contribution.

Cite this article: Prigitano A, et al. (2022). Neosartorya hiratsukae: Environmental isolation from intensive care units in an Italian hospital. Infection Control \& Hospital Epidemiology, 43: 949-950, https://doi.org/10.1017/ice.2021.136 antifungals were tested: itraconazole, voriconazole, posaconazole, isavuconazole and amphotericin B (Sigma-Aldrich, St Louis, MO).

Fungi grew from $18.2 \%$ of the environmental samples; molds grew from 8 samples. We identified 2 mold colonies, isolated from a cooling fan and from the handles of a patient bed, as A. niger and A. fumigatus. In total, 13 colonies of filamentous fungi isolated from 6 samples showed slow growth, with restricted white colonies and light-brown reverse. In particular, in one ICU, 12 colonies grew from the scialytic lamps ( $n=7)$ of 2 different patient beds, from the computer keyboard of the medical station $(n=3)$, from the patient vital signs monitor $(\mathrm{n}=1)$ and from the bed handles $(n=1)$. The last colony was isolated from the printer of the medical station of the other ICU. The microscopic examination of these colonies revealed A. fumigatus-like conidial head, a large number of cleistothecia, and hyaline, lenticular ascospores with equatorial crests. The $\beta$-tubulin sequences analysis showed a $100 \%$ homology with the reference sequence of $N$. hiratsukae (sequence AF057324).

Neosartorya hiratsukae antifungal susceptibility testing yielded the following MIC values: itraconazole, $0.12 \mathrm{mg} / \mathrm{L}$; voriconazole, $0.03 \mathrm{mg} / \mathrm{L}$; posaconazole, $0.06 \mathrm{mg} / \mathrm{L}$; isavuconazole, $0.12 \mathrm{mg} / \mathrm{L}$; and amphotericin B, $<0.03 \mathrm{mg} / \mathrm{L}$. No break points (BPs) have been specified for N. hiratsukae; however, the MIC values of our isolate are low and under the EUCAST BPs established for A. fumigatus. ${ }^{8}$

No cases of aspergillosis were diagnosed in the 2 ICUs in a 5-month period, 3 before the isolation of $N$. hiratsukae from the environment and 2 thereafter (Dr R. Grande, personal communication). In July 2020, we were able to perform further environmental samplings, but neither $N$. hiratsukae nor other fungi were isolated.

Like all filamentous fungi, Neosartorya also has an environmental origin. In the literature, $N$. hiratsukae has been reported in the indoor air ${ }^{1}$ and on drywall, where the small white colonies are hardly visible, so the spores can easily spread in the environment causing a health risk. ${ }^{9}$ In the 2 ICUs examined, no renovations had 
been made in the period prior to the sampling, and there is no drywall in these wards. However, the presence of this material in other wards or hospital common areas is not excluded. The presence of $N$. hiratsukae on the computer keyboard and printer in the medical stations suggests that it may have been spread by healthcare professionals.

Unfortunately, the rapid evolution of the COVID-19 epidemic in Italy shortly after our environmental sampling prevented further control samplings until 6 months later, when no N. hiratsukae were isolated. A more accurate sanitation during COVID-19 epidemic has probably reduced the presence of possible pathogens on the surfaces.

Despite the presence of $N$. hiratsukae in the environment of the sampled ICUs, no clinical isolates of $N$. hiratsukae were detected in the hospital in the same period, even if it cannot be ruled out due to the difficulties in identifying this species. Indeed, macroscopic and microscopic identification is not specific, and methods such as MALDI, in use in the hospital microbiology laboratories, do not identify this species. Neosartorya infections are probably underreported due to the aforementioned difficulties in identification and to the laboratory practice of discarding the nonsporulating or slowly sporulating white mycelia as laboratory contaminants.

Variable MIC values for N. hiratsukae are reported in the literature, showing mainly azole-susceptible patterns ${ }^{2,5,6}$ and some sporadic high itraconazole MIC values. ${ }^{6}$

Preventing nosocomial infections is very important, especially for ICU patients. Environmental control through environmental sampling of air and surfaces represents a valid tool that should be performed systematically, not only in the case of an outbreak. Laboratory routine methods for the identification are not sufficient to perform the correct identification of $N$. hiratsukae, obtainable only with molecular biology techniques. The correct identification of $N$. hiratsukae and other Neosartorya spp as well as their antifungal susceptibilities should be further investigated.
Acknowledgments. The authors would like to thank Dr Romualdo Grande for his communication and the hospital staff of the ICU involved in this study for their collaboration.

Financial support. No financial support was provided relevant to this article.

Conflicts of interest. All authors report no conflicts of interest relevant to this article.

\section{References}

1. Udagawa S, Tsubouchi H, Horie Y. Neosartorya hiratsukae, a new species of food-borne Ascomycetes. Trans Mycol Soc Jpn 1991;32:23-29.

2. Guarro J, Kallas EG, Godoy P, et al. Cerebral aspergillosis caused by Neosartorya hiratsukae, Brazil. Emerg Infect Dis 2002;8:989-991.

3. Predari SC, de Paulis AN, Veron D, Zucchini A, Santoianni JE. Fungal peritonitis in patients on peritoneal dialysis: twenty-five years of experience in a teaching hospital in Argentina. Rev Argent Microbiol 2007;39:213-217.

4. Shivaprakash MR, Jain N, Gupta S, Baghela A, Gupta A, Chakrabarti A. Allergic fungal rhinosinusitis caused by Neosartorya hiratsukae from India. Med Mycol 2009;47:317-320.

5. Mellado E, Alcazar-Fuoli L, Garcia-Effron G, Alastruey-Izquierdo A, Cuenca-Estrelal M, Rodriguez-Tudela JL. New resistance mechanisms to azole drugs in Aspergillus fumigatus and emergence of antifungal drugsresistant A. fumigatus atypical strains. Med Mycol 2006;44:S367-S371.

6. Koutroutsos K, Arabatzis M, Bougatsos G, Xanthaki A, Toutouza M, Velegraki A. Neosartorya hiratsukae peritonitis through continuous ambulatory peritoneal dialysis. J Med Microbiol 2010;59:862-865.

7. Rodriguez-Tudela JL, Arendrup MC, Arikan S, et al. European Committee on Antimicrobial Susceptibility Testing. EUCAST definitive document E.DEF 9.1: Method for Determination of Broth Dilution Minimum Inhibitory Concentrations of Antifungal Agents for Conidia-Forming Moulds. Växjö, Sweden: EUCAST; 2008.

8. Antifungal agents' breakpoint tables for interpretation of MICs-version 10.0. European Committee on Antimicrobial Susceptibility Testing website. https://www.eucast.org/fileadmin/src/media/PDFs/EUCAST_files/AFST/ Clinical_breakpoints/AFST_BP_v10.0_200204.pdf. Accessed March 25, 2021.

9. Andersen B, Dosen I, Lewinska AM, Nielsen KF. Pre-contamination of new gypsum wallboard with potentially harmful fungal species. Indoor Air 2017; 27:6-12.

\title{
Coronavirus disease 2019 (COVID-19) vaccination in healthcare workers: An early real-world experience
}

\author{
Andrew P. Jameson MD, FACP ${ }^{1,2}$ (1), Tara Sebastian BS $^{1}$ and Liberty R. Jacques DNP, RN, CIC \\ ${ }^{1}$ Michigan State University College of Human Medicine, Grand Rapids, Michigan and ${ }^{2}$ Mercy Health Saint Mary's Hospital, Grand Rapids, Michigan
}

To the Editor-The spread of the novel severe acute respiratory syndrome coronavirus 2 (SARS-CoV-2) and its associated coronavirus disease (COVID-19) has affected millions of people worldwide. As of February 2021, the pandemic had claimed the lives of $>2.4$ million individuals. ${ }^{1}$ Highly effective vaccines against the SARS-CoV-2 virus have attracted intense attention as an attainable solution to mitigate further spread.

\footnotetext{
Author for correspondence: Andrew P. Jameson, E-mail: andrew.jameson@mercyhealth. com

Cite this article: Jameson AP, Sebastian T, and Jacques LR. (2022). Coronavirus disease 2019 (COVID-19) vaccination in healthcare workers: An early real-world experience. Infection Control \& Hospital Epidemiology, 43: 950-951, https://doi.org/10.1017/ ice. 2021.171
}

One such vaccine is the BNT162b2 mRNA vaccine, first described by Polack et al, ${ }^{2}$ now being produced and distributed by Pfizer (New York City, NY). In December 2020, data from phase 2 and 3 clinical trials with 36,523 participants were published describing the initial efficacy of the vaccine in preventing symptomatic COVID-19 transmission. These data suggested that, after 1 dose, the BNT162b2 vaccine had an efficacy of $52 \%$. At 7 days after the second dose, the vaccine showed a highly promising efficacy of $95 \%{ }^{3}$

Here, we present data from a vaccination program for healthcare workers (HCWs) of a medium-sized urban hospital in the Midwest region in the United States. Throughout the vaccination effort, the community burden of COVID-19 remained concerningly high, with 